\title{
Cross-talk of healthy and impaired human tissues for dissection of disease pathogenesis
}

Alice Zoso ${ }^{1,2, *}$, Alessandro Zambon ${ }^{1,2, *}$, Onelia Gagliano ${ }^{1,2}$, Stefano Giulitti ${ }^{1,2}$, Luca Prevedello ${ }^{3}$, Gian Paolo Fadini ${ }^{4}$, Camilla Luni ${ }^{5}$, Nicola Elvassore ${ }^{1,2,5,6, \#}$

${ }^{1}$ Venetian Institute of Molecular Medicine, Via Orus 2, 35131 Padova, Italy

${ }^{2}$ Department of Industrial Engineering, University of Padova, Via Marzolo 9, 35129 Padova, Italy

${ }^{3}$ Department of Surgery, Oncology and Gastroenterology, University of Padova, 35124 Padova, Italy

${ }^{4}$ Department of Medicine, University of Padova, 31128 Padova, Italy

${ }^{5}$ Shanghai Institute for Advanced Immunochemical Studies, ShanghaiTech University, 393 Huaxia Road, Shanghai 201210, China

${ }^{6}$ Stem Cells \& Regenerative Medicine Section, UCL Great Ormond Street Institute of Child Health, 30 Guilford Street, London WC1N 1EH, UK

*These authors equally contributed to this work.

${ }^{\#}$ Corresponding author:

Nicola Elvassore

E-mail: n.elvassore@ucl.ac.uk

Stem Cells \& Regenerative Medicine Section,

UCL Great Ormond Street Institute of Child Health,

30 Guilford Street, London WC1N 1EH, UK

Telephone: +390498275469

Fax: +39049827 7599

Keywords: microfluidics, tissue-on-chip, tissue cross-talk. 


\begin{abstract}
Systemic diseases affect multiple tissues that interact with each other within a network difficult to explore at the body level. However, understanding the inter-dependences between tissues could be of high relevance for drug target identification, especially at the first stages of disease development. In vitro systems have the advantages of accessibility to measurements and precise controllability of culture conditions, but currently have limitations in mimicking human in vivo systemic tissue response.
\end{abstract}

In this work, we present an in vitro model of cross-talk between an ex vivo culture of adipose tissue from an obese donor and a skeletal muscle in vitro model from a healthy donor. This is relevant to understand type 2 diabetes mellitus pathogenesis, as obesity is one of its main risk factors. The human adipose tissue biopsy was maintained as a three-dimensional culture for 48 hours. Its conditioned culture medium was used to stimulate a human skeletal muscle-on-chip, developed by differentiating primary cells of a patient's biopsy under topological cues and molecular selfregulation. This system has been characterized to demonstrate its ability to mimic important features of the normal skeletal muscle response in vivo. We then found that the conditioned medium from a diseased adipose tissue is able to perturb the normal insulin sensitivity of a healthy skeletal muscle, as reported in the early stages of diabetes onset.

In perspective, this work represents an important step towards the development of technological platforms that allow to study and dissect the systemic interaction between unhealthy and healthy tissues in vitro. 


\section{Introduction}

Systemic diseases disrupt the overall patient physiology, involving changes in multiple tissues ${ }^{1}$. Studying how they develop is a difficult task due to the high interconnectivity of the body system. For example, through blood circulation, endocrine signals from one tissue affect the physiological function of other tissues, even if remotely located.

In vivo, dissecting the cross-talk between tissues is complicated both by the difficulty to access human tissues and by the interplay between tissues, which prevents understanding the directionality of the phenomena. However, understanding which tissue is the most relevant target for therapeutic intervention is critical, especially during disease pathogenesis.

On the contrary, in vitro culture systems offer the advantages of controlled manipulation of experimental conditions for dissecting tissue-to-tissue communication and of accessibility to measurements ${ }^{2}$. The most used in vitro models to study the cross talk between different tissues are based on no-contact co-culture systems, called transwell, where different cell types are cultured on the same dish sharing the same culture medium, but physically separated by a porous membrane. One limitation of these systems is that they cannot be used to understand the directionality of the interactions. Another strategy commonly used is to culture each cell type in different dishes, and to mimic their interaction by putting the medium used to culture one type of cells on the other cell type. The conditioned medium from the "upstream" cells will contain their secreted proteins, and will be used to understand the effect on the "downstream" cells in absence of a returning feedback ${ }^{3}$. However, aiming at studying the cross-talk between tissues, the first requirement and current limitation in the field is that the in vitro tissue culture has to accurately mimic the relevant features of the tissue in vivo, in order to achieve an in vivo-like response. So called tissues-on-chip are systems that integrate cells within microfluidic devices that promote their organization to mimic tissue functionality ${ }^{4-6}$. Miniaturization has been shown to be critical to favor the development of tissue-like structures in vitro, because the confined environment preserves the scale ratio of 
different molecular transport phenomena occurring during the reciprocal interaction between cells and surrounding microenvironment within tissues in vivo ${ }^{7}$

Moreover, microfluidics has several other advantages. The small volume requires only few cells, and make possible to obtain multiple parallel cultures from a single tissue biopsy. Considering the limited access to human biopsies of tissues, especially from healthy patients, this aspect is very critical for in vitro studies of human biology. Another advantage of microfluidics is that it requires a little amount of medium for cell culture, few microliters instead of multiple milliliters, thus the same amount of conditioned medium from one tissue can be used to perturb the culture of many other target tissues.

Up to now, multiple tissues-on-chip have been developed ${ }^{4,7}$, although the application of these technologies to human primary cells is more limited ${ }^{5,8,9}$. Some studies have been performed to understand the interaction between different tissues in vitro, but mainly cell lines have been used so far, as recently reviewed ${ }^{2,10}$.

In this work, we study the cross-talk between adipose and skeletal muscle tissues using human primary cell sources, within the context of metabolic impairments. These two tissues are the main responsible for glucose uptake from the blood stream in response to insulin after meals ${ }^{11}$. Their reciprocal interaction is strictly linked to the delicate equilibrium between diet and physical exercise, object of many studies especially at the organism level ${ }^{12,13}$. Because obesity is known to be an important risk factor for the development of type 2 diabetes ${ }^{14,15}$, here we study the effect of an adipose tissue from an obese non-diabetic patient onto a skeletal muscle from a healthy non-obese patient, in terms of perturbations of insulin signaling (Figure 1).

Towards this aim, we developed the first human in vitro model of skeletal muscle on a chip. In previous works a skeletal muscle cell line was used to develop a mouse model on chip ${ }^{16-18}$. We have previously demonstrated that inducing an aligned topology on a dish culture promotes human skeletal muscle-like functionality ${ }^{19,20}$. Here, we extend these results to build a human skeletal muscle-on-chip, with an innovative technology to produce a cell-adhesive pattern in microfluidics. 
We obtained human adipose tissue as a three-dimensional biopsy, preserving the in vivo organ structure, and we cultured it in vitro for only 48 hours to obtain the conditioned medium for the cross-talk experiments. The data obtained showed the possibility to dissect in vitro the role of an impaired adipose tissue onto the insulin sensitivity of a healthy skeletal muscle.

In vitro studies of human tissue cross-talk are still very limited, but will be more and more relevant for understanding tissue interactions at the cellular level in the future. The possibility to use stem cells to develop tissues-on-chip will greatly favor these studies, given the current difficulty of collecting primary human tissue biopsies ${ }^{7}$. In perspective, the use of microtechnologies, coupled with stem cell-derived tissues, will make possible not only to develop reliable in vitro models of human tissues, but also to understand the complexity of the whole body physiology by dissecting their interactions.

\section{Materials and Methods}

\section{Microfluidic devices}

The microfluidic devices used for culturing skeletal muscle cells were produced according to standard lithographic techniques $^{21}$. The design of the devices was previously described ${ }^{16}$.

\section{Surface micro-pattern within microfluidic culture chambers}

Selected areas of the culture surface in the microfluidic chambers were made cell repellent by photo-patterning of linear acrylamide (Figure 2a). After fabrication, microfluidic chambers were rinsed with isopropanol and distilled water, and treated with a $0.1 \mathrm{M} \mathrm{NaOH}$ solution for $1 \mathrm{~h}$. A $0.3 \%$ solution of 3-acryloxypropil methacrylate (Acros) in ethanol and 5\% v/v acetic acid was used to treat oxidized glass surface inside each microfluidic channel. After $5 \mathrm{~min}$, channels were rinsed twice with ethanol and filled with an acrylamide solution (8\% v/v acrylamide (Sigma-Aldrich), 50 mM HEPES, 10\% v/v methanol solution with 35 mg/mL Irgacure 2959 (Ciba)). Then, microfluidic chips were placed on a transparency mask, printed with a pattern of $300-\mu \mathrm{m}$ wide parallel lanes, and 
exposed for 2 min to UV light, generated by a collimated 50-W UV-lamp (Dymax Bluewave 50W). Unreacted material was removed by rinsing the channels twice with distilled water. Sterilization was performed by UV-light exposure for $20 \mathrm{~min}$. Finally, a $4^{\circ} \mathrm{C}$-cold Matrigel (Becton-Dickinson) solution, $2.5 \%$ in Dulbecco's Modified Eagle Medium (DMEM), was injected into the microchannels and incubated for $1 \mathrm{~h}$ at room temperature, producing a patterned cell-adhesive surface coating, due to linear acrylamide protein exclusion.

\section{Human primary myoblast extraction and expansion}

Human primary myoblasts were kindly provided by Dr. Karim Bouzakri (University of Geneva), isolated as previously described ${ }^{22}$ from a skeletal muscle biopsy of non-diabetic patients undergoing surgery after informed consent by Ethics Committee. Briefly, after explant, the human skeletal muscle biopsy was maintained in $20 \mathrm{~mL}$ of physiological solution at $+4^{\circ} \mathrm{C}$ for $2 \mathrm{~h}$ to allow blood coagulation. It was then processed under sterile hood with a scalpel, to isolate single muscle fibers from connective, adipose, and blood vessel tissues. The isolated fibers were maintained in a solution $0.05 \%$ Trypsin-EDTA at $37^{\circ} \mathrm{C}$ for $3 \mathrm{~h}$ to extract single-cell myoblast precursors. The surnatant cell suspension was then transferred into a new tube, added of an equal amount of fetal calf serum (FCS, Life Technologies), and centrifuged at $200 \mathrm{~g}$ for $5 \mathrm{~min}$. The pellet was resuspended in $10 \mathrm{~mL}$ of skeletal muscle cell basal medium (PromoCell), and pre-plated in a bacterial petri dish for $2 \mathrm{~h}$, the time required to have fibroblast adhesion and therefore exclusion. Afterwards, medium with myoblasts still in suspension was collected and myoblasts plated in a 25$\mathrm{mm}^{2}$ tissue culture flask. Human primary myoblasts were cultured for at least 7 days, with medium changes every 2-3 days. Cells were split before confluence by Trypsin-EDTA $0.05 \%$.

\section{Human myoblast microfluidic integration and differentiation}

After Matrigel coating, microfluidic chambers were rinsed with skeletal muscle cell basal medium. A myoblast suspension was injected into the chambers for seeding at a final density of 200 
cell $/ \mathrm{mm}^{2}$. The microfluidic devices were then placed in a biological incubator at $37^{\circ} \mathrm{C}$ and $5 \% \mathrm{CO}_{2}$. Medium was changed every $12 \mathrm{~h}$. At cell confluence, differentiation of human myoblasts into myotubes was induced by switching to a differentiation medium, composed of DMEM with $2 \%$ horse serum (HS, Life Technologies). Thereafter, medium was changed every $24 \mathrm{~h}$ for 6-8 days.

\section{Immunofluorescence analysis}

For immunofluorescence analysis, myotubes were fixed in $4 \%(\mathrm{w} / \mathrm{v})$ paraformaldehyde (PFA, Sigma-Aldrich) for $15 \mathrm{~min}$, and stained with primary antibodies in PBS with $3 \%$ bovine serum albumin (BSA, Sigma-Aldrich) and $0.25 \%$ (v/v) Tryton-X-100 (Sigma-Aldrich). Primary antibodies: Myosin Heavy Chain II 1:100 (MHC, Sigma-Aldrich) and Desmin 1:100 (DAKO). Alexa488 and Alexa594 conjugated mouse secondary antibodies (1:200) were used (Life Technologies). Nuclei were stained with Hoechst 33342 (Life Technologies). Images were acquired with a DMI6000B epifluorescence microscope (Leica Microsystems) and with a TCS SP5 confocal microscope (Leica Microsystems).

\section{Human adipose tissue isolation and culture}

A biopsy of a visceral adipose tissue was collected from an obese individual undergoing surgery (sleeve gastrectomy) at the University Hospital of Padova (Italy), after informed consent. Collection and storage of biological samples was approved by the local Ethical Committee. After isolation, the biopsy was maintained in $5 \mathrm{~mL}$ of DMEM with 5-mM glucose (Life Technologies) for up to $3 \mathrm{~h}$ at room temperature. Then, it was washed with phosphate buffer saline (PBS, Life Technologies) and cut into 4-mm diameter slices with the aid of a sterile biopsy punch (Miltex) and a scalpel. Each slice was put in a 48-well plate with $300 \mu \mathrm{L}$ of 5-mM glucose DMEM. Conditioned media were collected after $48 \mathrm{~h}$ of culture and stocked at $-20^{\circ} \mathrm{C}$ until use. These media were used as conditioned media for the stimulation of human myotubes in microfluidics. 


\section{Tissue cross-talk experiments}

After myoblast differentiation into myotubes in the patterned microfluidic culture chambers, medium was changed to DMEM (5mM glucose) the night before the stimulation. Next, adipose tissue-conditioned media were used for a 24-h pre-conditioning; control chambers were kept in DMEM (5mM glucose), with or without TNF $\alpha(20 \mathrm{ng} / \mathrm{mL})$. At the end of the $24 \mathrm{~h}$, a $10-\mathrm{min}$ stimulation was performed using DMEM with or without insulin $(100 \mathrm{nM})$. The whole experiment was performed using one adipose tissue and two skeletal muscle biopsies. Next, the microfluidic chambers were washed with cold PBS before lysate collection for Western blot analysis.

\section{Western blot analysis}

Microfluidic cultures were treated with $12-\mu \mathrm{L}$ ice-cold lysis buffer: $5 \%$ deoxycholic acid (DOC, Sigma-Aldrich) in Tris-Buffered Saline (TBS), supplemented with Complete EDTA-free protease inhibitor (Roche) and anti-phosphatase cocktail (Sigma-Aldrich). Lysis buffer was directly injected in each microfluidic chamber and whole cell lysate collected. PAGE was performed with 4-12\% NuPAGE polyacrylamide gels and MOPS buffer (Life Technologies). Proteins were blotted on PVDF membranes (Life Technologies) and detected with Carestream films (Kodak). A 1:1000 dilution of primary antibodies for Phospho-Akt (Ser 473), Akt, Phospho-AS160 (Thr 642), AS160 (all from Cell Signaling), IRS-1 (Millipore), GAPDH (Abcam), and HRP-conjugated secondary antibodies (mouse, Bio-Rad; rabbit, Life Technologies) were used. Gel images were quantified by standard image analysis, and ANOVA was performed to identify statistically significant differences.

\section{Results and Discussion}

Micro-pattern integration in the microfluidic chip

The importance of topology in human skeletal muscle differentiation has been previously reported $^{19,20,23}$. In particular, a substrate for myoblast culture with an elongated geometry pattern 
favors the alignment of differentiating myotubes and improves their functional maturation. In addition, performing myotube differentiation within microfluidic devices makes possible to take advantage of some strengths of this technology: specifically, the small volume and ease of parallelization. These features make microfluidics particularly attractive for performing screening studies starting with a small number of cells, and using a small amount of medium to test several conditions.

Micropatterning topology is difficult to integrate within microfluidic cell cultures. Few solutions were previously proposed and are described next. Folch's group performed a cell adhesive pattern on a culture substrate before attaching the microfluidic device on it $^{17}$; however, the subsequent assembly of the microfluidic chip cannot be performed by plasma bonding as usual, and becomes more unpractical and less robust. More recently, micropatterning by pneumatic microfluidics have been suggested ${ }^{24}$. However, this method requires multi-layer microfluidics coupled with a pneumatic system for controlling the production stages, and the whole design and production have to be changed in case of variations in the pattern geometry.

We successfully produced a defined pattern of cell adhesion directly inside the assembled microfluidic chip (Figure 2a). To topologically control cell-adhesive and repellent areas, linear acrylamide was photo-patterned according to the design of a photomask, placed between the acrylamide solution in the microfluidic chambers and a UV light source. This method is rapid and practical, and the pattern geometry can be easily changed by simply printing different designs in the photomask. After washing and sterilization, cells are seeded in the microfluidic chambers and only adhere where the surface is free of the linear acrylamide pattern, with a resulting specific topology in culture.

For this study, a pattern of $300-\mu \mathrm{m}$ wide lanes was produced. The width of the lanes was optimized in our previous works ${ }^{19,20}$, which demonstrated that this width represents a trade-off between the accumulation of endogenous cell-secreted signals, which requires cell crowding, and the geometric effect for the myotube alignment, which is better promoted with thinner lanes. 


\section{Human skeletal muscle culture and characterization in the microfluidic chip}

Human primary myoblasts were seeded and differentiated in the patterned microfluidic chambers. Cells were seeded on Matrigel, which introduces some uncertainty in the system due to the undefined and complex composition. However, during the 2-week cell culture before the perturbation experiments with conditioned media, cells deposited and remodeled their endogenous extracellular matrix and the effect of the original coating becomes less relevant for the purpose of the study. The protocol includes two stages: first, cells are cultured in a skeletal muscle basal medium until they reach confluence (after $\sim 4$ days from seeding); then, the medium is changed to one containing factors promoting differentiation and fusion of myoblasts into myotubes. The cells were cultured in the microfluidic device for up to 14 days. A longer culture period was prevented by myotube detachment due to spontaneous contractions.

Aside of medium composition, the culture conditions needed an optimized frequency of medium change. As we previously demonstrated, in microscale cultures the balance between exogenous factors introduced with the medium and endogenous cell-secreted factors is very critical in modifying cellular response ${ }^{8,25}$. Conversely, conventional culture systems are quite insensitive to modifications to the frequency of medium change, as long as cell starvation is avoided, because cell-secreted factors are rapidly diluted in the large medium volume. The accumulation of cellsecreted factors occurring in microfluidics could be seen as a criticality because makes the system over-sensitive, but it actually produces an increase in endogenous factor concentrations to the point that their role becomes biologically relevant, mimicking the self-regulation occurring within tissues in vivo. In this work, the following optimal frequencies of medium change were obtained: medium changes every 12 hours during the first stage of cell proliferation, and every 24 hours during the second stage of myotube differentiation. These conditions proved optimal to balance the higher demand of nutrients during the first proliferative stage, and the need of promoting cell selfregulation during differentiation. 
The morphological progression during proliferation and differentiation on the microfluidic pattern with the optimized protocol is shown in Figure 2b. At day 1 from seeding, cells are proliferating only within the adhesive surface of the pattern. After 4 days cells already show some alignment along the longitudinal direction. At day 8, elongated myotubes are visible, parallel to the lanes of the pattern.

Myotubes were further characterized by immunofluorescence assay for specific skeletal muscle proteins: Desmin and Myosin Heavy Chain (MHC), together with nuclei staining. These two proteins are components of the contraction machinery of the skeletal muscle, thus related to the main tissue functionality in vivo. Figure 3 shows myotubes as elongated multinucleated cells formed by the fusion of myoblasts. Immunofluorescence analysis detected the expression of both Desmin and MHC. As highlighted in Figure 3d, within our in vitro system, Desmin is not only expressed, but also properly localized within myotubes according to a regular pattern typical of a functional striated muscle.

Overall, this characterization shows the in vitro maturation of myotubes in microfluidics, according to multiple features characterizing the in vivo skeletal muscle. Thus, the in vitro system we developed could be a reliable model for studies of skeletal muscle response under external stimuli.

\section{Adipose tissue and skeletal muscle cross-talk}

Skeletal muscle is the tissue responsible for most of glucose uptake from the blood stream ${ }^{11}$. Insulin signaling pathway within muscle cells is a complex network of protein interactions that is activated in response to the presence of high concentrations of extra-cellular insulin, which is produced by the pancreas $^{26}$. In this work, we focus on the specific sub-network of the insulin signaling pathway shown in Figure 4a, where, after insulin binding upon its receptor, a cascade of protein phosphorylation events is generated, ultimately leading to the translocation of glucose transporter GLUT4 to the cell membrane. Once it reaches the cell surface, GLUT4 plays as a channel for glucose entrance into the cell. Thus, this sub-network links extracellular insulin concentration to 
cell glucose uptake. To understand the impact of unidentified signaling molecules secreted by an obese adipose tissue on skeletal muscle insulin-stimulated glucose uptake, we pinpointed the targets within this sub-network by means of Western blot analysis: specifically, IRS1, and Akt and AS160 both in their unphosphorylated and phosphorylated states.

Once myotubes had reached their functional maturation within the chip, they were cultured in adipose tissue-conditioned medium for 24 hours and then stimulated, or not, for 10 min with an insulin concentration physiologically sufficient to activate the pathway. After this stimulus, the culture was stopped, cells lysed, and cell protein content from each microfluidic culture chamber collected for quantification of selected proteins. We compared these results with a negative control given by cells stimulated, or not, with insulin without prior exposure to adipose tissue-conditioned medium. We also compared these results with a commonly used positive control given by cells stimulated, or not, with insulin after been exposed to a medium containing TNFa, which is a protein, produced also by the adipose tissue especially in obese patients, known to induce in vitro insulin impaired response on healthy myotubes ${ }^{27}$. Thanks to the small number of cells required in each microfluidic platform, cells from the same patient could be used to test all these conditions in parallel.

The results of protein quantification at the end of the experiment are reported in Figure 4b-d. First, in the negative control condition, insulin stimulation does not affect IRS1 total content, and strongly induces Akt and AS160 phosphorylation. Thus, the in vitro skeletal muscle model we developed reproduces the known features of a normal insulin response ${ }^{26}$.

Second, in the positive control, TNF $\alpha$ does not affect the total IRS1 content (Figure 4b). IRS1 expression is known to have reduced expression in obese patients ${ }^{28}$. This result may point out the limitations related to $\mathrm{TNF} \alpha$ use for mimicking the complexity of adipose tissue-skeletal muscle cross-talk in obesity. On the contrary, TNFa significantly decreases insulin-induced Akt phosphorylation (Figure 4c); however, despite reduced activation of Akt, AS160 phosphorylation is comparable to the negative control in this condition (Figure 4d). Due to the complexity of the 
overall insulin signaling network, it is not possible to discriminate between two possible scenarios: (i) the reduced activation of Akt is still not limiting for AS160 phosphorylation, and (ii) the network is redundant and another kinase can phosphorylate AS160 compensating for Akt reduced activity, as it occurs when AS160 is phosphorylated by stimuli other than insulin ${ }^{29}$. This result is in contrast with another study where TNF $\alpha$ was shown to decrease AS160 phosphorylation; however, due to the very different experimental design and duration of the stimulation, it is difficult to have a direct comparison between the two studies ${ }^{30}$.

Last, adipose tissue-conditioned medium more strongly disrupts skeletal muscle insulin response: IRS 1 content is approximately halved (Figure 4b), and both Akt and AS160 show a limited response to insulin in terms of fraction of phosphorylated protein, while the basal (non insulin stimulated) phosphorylated fraction is comparable in the three conditions with different preconditioning (Figure 4c-d). The comparable phosphorylation level of Akt between the adipose tissue-conditioned medium and $\mathrm{TNF} \alpha$ containing medium, seems to support the second scenario suggested above, and we can speculate that, because multiple signals are secreted by the adipose tissue, the impact on the insulin signaling network occurs at different nodes, disrupting the redundancy of its functionality ${ }^{31,32}$.

Taken together, these results show that the developed skeletal muscle-on-chip can accurately reproduce the normal activation of the insulin pathway. Furthermore, they also highlight differences in pathway impairments due to $\mathrm{TNF} \alpha$, commonly used to in vitro study insulin impaired response, and due to a more complex, although uncharacterized, mixture of signaling secreted by an adipose tissue derived from an obese patient.

\section{Conclusions}

In this study we reported the first in vitro human skeletal muscle-on-chip model. We characterized it morphologically, biochemically, and functionally, demonstrating its ability to respond to insulin stimulation. Besides the biological reliability of this model, miniaturization also decreases the 
requirements in terms of number of cells and amount of conditioned medium, favoring throughput and parallelization of experiments. Due to the high variability of biological systems, a design of experiments that tests several conditions on the same biopsy strongly enhances the statistical significance of the results.

In perspective, we envision that tissue-on-chips will be more and more used to bring physiological studies from the patient to the laboratory bench, in terms of tissue cross-talk. In vitro systems expand the parameter space of possible investigations respect to in vivo studies, helping the dissection of biological mechanisms. For example, here we could show the interaction between an adipose tissue from an obese patient and a skeletal muscle from a healthy patient, comparing this to the same tissue not subjected to the pathological perturbation. This kind of tissue cross-talk studies could be relevant in understanding disease pathogenesis with high accuracy at the molecular level, and ultimately developing tissue-targeted therapies ${ }^{6}$.

\section{Acknowledgements}

The authors would like to thank Dr. Karim Bouzakri at Geneva University for providing human myoblasts. This research was supported by the European Foundation for the Study of Diabetes (EFSD, Albert Reynold Travel Fellowships to A. Zoso), ShanghaiTech University, University of

Padova (TRANSAC and PRAT), CaRiPaRo Foundation, Telethon Foundation (GGP15275), and Oak Foundation Award W1095/OCAY-14-191.

\section{Literature Cited}

1. Gancheva S, Jelenik T, Álvarez-Hernández E, Roden M. Interorgan Metabolic Crosstalk in Human Insulin Resistance. Physiol Rev. 2018;98(3):1371-1415. doi:10.1152/physrev.00015.2017.

2. Lee SH, Sung JH. Organ-on-a-Chip Technology for Reproducing Multiorgan Physiology. Adv Healthc Mater. 2017;7(2):1700419. doi:10.1002/adhm.201700419.

3. Lam YY, Janovská A, McAinch AJ, Belobrajdic DP, Hatzinikolas G, Game P, Wittert GA. The use of adipose tissue-conditioned media to demonstrate the differential effects of fat depots on 
insulin-stimulated glucose uptake in a skeletal muscle cell line. Obes Res Clin Pract. 2011;5(1):e43-e54. doi:10.1016/j.orcp.2010.12.002.

4. Luni C, Serena E, Elvassore N. Human-on-chip for therapy development and fundamental science. Curr Opin Biotechnol. 2014;25:45-50. doi:10.1016/j.copbio.2013.08.015.

5. Esch EW, Bahinski A, Huh D. Organs-on-chips at the frontiers of drug discovery. Nat Rev Drug Discov. 2015;14(4):248-260. doi:10.1038/nrd4539.

6. Neuži P, Giselbrecht S, Länge K, Huang TJ, Manz A. Revisiting lab-on-a-chip technology for drug discovery. Nat Rev Drug Discov. 2012;11(8):620-632. doi:10.1038/nrd3799.

7. Gagliano O, Elvassore N, Luni C. Microfluidic technology enhances the potential of human pluripotent stem cells. Biochem Biophys Res Commun. 2016;473(3):683-687. doi:10.1016/j.bbrc.2015.12.058.

8. Giobbe GG, Michielin F, Luni C, Giulitti S, Martewicz S, Dupont S, Floreani A, Elvassore N. Functional differentiation of human pluripotent stem cells on a chip. Nat Methods. 2015;12(7):637. doi:10.1038/nmeth.3411.

9. Ronaldson-Bouchard K, Vunjak-Novakovic G. Organs-on-a-Chip: A Fast Track for Engineered Human Tissues in Drug Development. Cell Stem Cell. 2018;22(3):310-324. doi:10.1016/j.stem.2018.02.011.

10. Abaci HE, Shuler ML. Human-on-a-chip design strategies and principles for physiologically based pharmacokinetics/pharmacodynamics modeling. Integr Biol. 2015;7(4):383-391. doi:10.1039/c4ib00292j.

11. Peppa M, Koliaki C, Nikolopoulos P, Raptis SA. Skeletal Muscle Insulin Resistance in Endocrine Disease. J Biomed Biotechnol. 2010;2010. doi:10.1155/2010/527850.

12. Pedersen BK. The diseasome of physical inactivity - and the role of myokines in muscle-fat cross talk. J Physiol. 587(23):5559-5568. doi:10.1113/jphysiol.2009.179515.

13. Smith U, Kahn BB. Adipose tissue regulates insulin sensitivity: role of adipogenesis, de novo lipogenesis and novel lipids. J Intern Med. 280(5):465-475. doi:10.1111/joim.12540.

14. Kusminski CM, Bickel PE, Scherer PE. Targeting adipose tissue in the treatment of obesityassociated diabetes. Nat Rev Drug Discov. 2016;15(9):639-660. doi:10.1038/nrd.2016.75.

15. Czech MP. Insulin action and resistance in obesity and type 2 diabetes. Nat Med. 2017;23(7):804-814. doi:10.1038/nm.4350.

16. Zambon A, Zoso A, Luni C, Frommer WB, Elvassore N. Determination of glucose flux in live myoblasts by microfluidic nanosensing and mathematical modeling. Integr Biol. 2014;6(3):277288. doi:10.1039/C3IB40204E.

17. Tourovskaia A, Figueroa-Masot X, Folch A. Differentiation-on-a-chip: A microfluidic platform for long-term cell culture studies. Lab Chip. 2005;5(1):14-19. doi:10.1039/B405719H.

18. Shimizu K, Araki H, Sakata K, Tonomura W, Hashida M, Konishi S. Microfluidic devices for construction of contractile skeletal muscle microtissues. J Biosci Bioeng. 2015;119(2):212-216. doi:10.1016/j.jbiosc.2014.07.003. 
19. Serena E, Zatti S, Reghelin E, Pasut A, Cimetta E, Elvassore N. Soft substrates drive optimal differentiation of human healthy and dystrophic myotubes. Integr Biol Quant Biosci Nano Macro. 2010;2(4):193-201. doi:10.1039/b921401a.

20. Zatti S, Zoso A, Serena E, Luni C, Cimetta E, Elvassore N. Micropatterning topology on soft substrates affects myoblast proliferation and differentiation. Langmuir ACS J Surf Colloids. 2012;28(5):2718-2726. doi:10.1021/la204776e.

21. Qin D, Xia Y, Whitesides GM. Soft lithography for micro- and nanoscale patterning. Nat Protoc. 2010;5(3):491-502. doi:10.1038/nprot.2009.234.

22. Bouzakri K, Roques M, Gual P, Espinosa S, Guebre-Egziabher F, Riou J-P, Laville M, Marchand-Brustel YL, Tanti J-F, Vidal H. Reduced activation of phosphatidylinositol-3 kinase and increased serine 636 phosphorylation of insulin receptor substrate-1 in primary culture of skeletal muscle cells from patients with type 2 diabetes. Diabetes. 2003;52(6):1319-1325. doi:10.2337/diabetes.52.6.1319.

23. Choi JS, Lee SJ, Christ GJ, Atala A, Yoo JJ. The influence of electrospun aligned poly( $\varepsilon$ caprolactone)/collagen nanofiber meshes on the formation of self-aligned skeletal muscle myotubes. Biomaterials. 2008;29(19):2899-2906. doi:10.1016/j.biomaterials.2008.03.031.

24. Wang J-C, Liu W, Tu Q, Ma C, Zhao L, Wang Y, Ouyang J, Pang L, Wang J. High throughput and multiplex localization of proteins and cells for in situ micropatterning using pneumatic microfluidics. Analyst. 2015;140(3):827-836. doi:10.1039/C4AN01972E.

25. Giulitti S, Magrofuoco E, Prevedello L, Elvassore N. Optimal periodic perfusion strategy for robust long-term microfluidic cell culture. Lab Chip. 2013;13(22):4430-4441. doi:10.1039/C3LC50643F.

26. Taniguchi CM, Emanuelli B, Kahn CR. Critical nodes in signalling pathways: insights into insulin action. Nat Rev Mol Cell Biol. 2006;7(2):85-96. doi:10.1038/nrm1837.

27. Imai Y, Dobrian AD, Weaver JR, Butcher MJ, Cole BK, Galkina EV, Morris MA, Taylor-Fishwick DA, Nadler JL. Interaction between cytokines and inflammatory cells in islet dysfunction, insulin resistance and vascular disease. Diabetes Obes Metab. 15(s3):117-129. doi:10.1111/dom.12161.

28. Goodyear LJ, Giorgino F, Sherman LA, Carey J, Smith RJ, Dohm GL. Insulin receptor phosphorylation, insulin receptor substrate-1 phosphorylation, and phosphatidylinositol 3kinase activity are decreased in intact skeletal muscle strips from obese subjects. J Clin Invest. 1995;95(5):2195-2204. doi:10.1172/JCI117909.

29. Kramer HF, Witczak CA, Fujii N, Jessen N, Taylor EB, Arnolds DE, Sakamoto K, Hirshman MF, Goodyear LJ. Distinct Signals Regulate AS160 Phosphorylation in Response to Insulin, AICAR, and Contraction in Mouse Skeletal Muscle. Diabetes. 2006;55(7):2067-2076. doi: $10.2337 / \mathrm{db} 06-0150$.

30. Plomgaard P, Bouzakri K, Krogh-Madsen R, Mittendorfer B, Zierath JR, Pedersen BK. Tumor Necrosis Factor- $\alpha$ Induces Skeletal Muscle Insulin Resistance in Healthy Human Subjects via Inhibition of Akt Substrate 160 Phosphorylation. Diabetes. 2005;54(10):2939-2945. doi:10.2337/diabetes.54.10.2939. 
31. Luni C, Shoemaker JE, Sanft KR, Petzold LR, Doyle FJ. Confidence from uncertainty - A multi-target drug screening method from robust control theory. Bmc Syst Biol. 2010;4. doi:10.1186/1752-0509-4-161.

32. Luni C, Doyle FJ. Robust multi-drug therapy design and application to insulin resistance in type 2 diabetes. Int J Robust Nonlinear Control. 2011;21(15):1730-1741. doi:10.1002/rnc. 1756. 


\section{FIGURES}

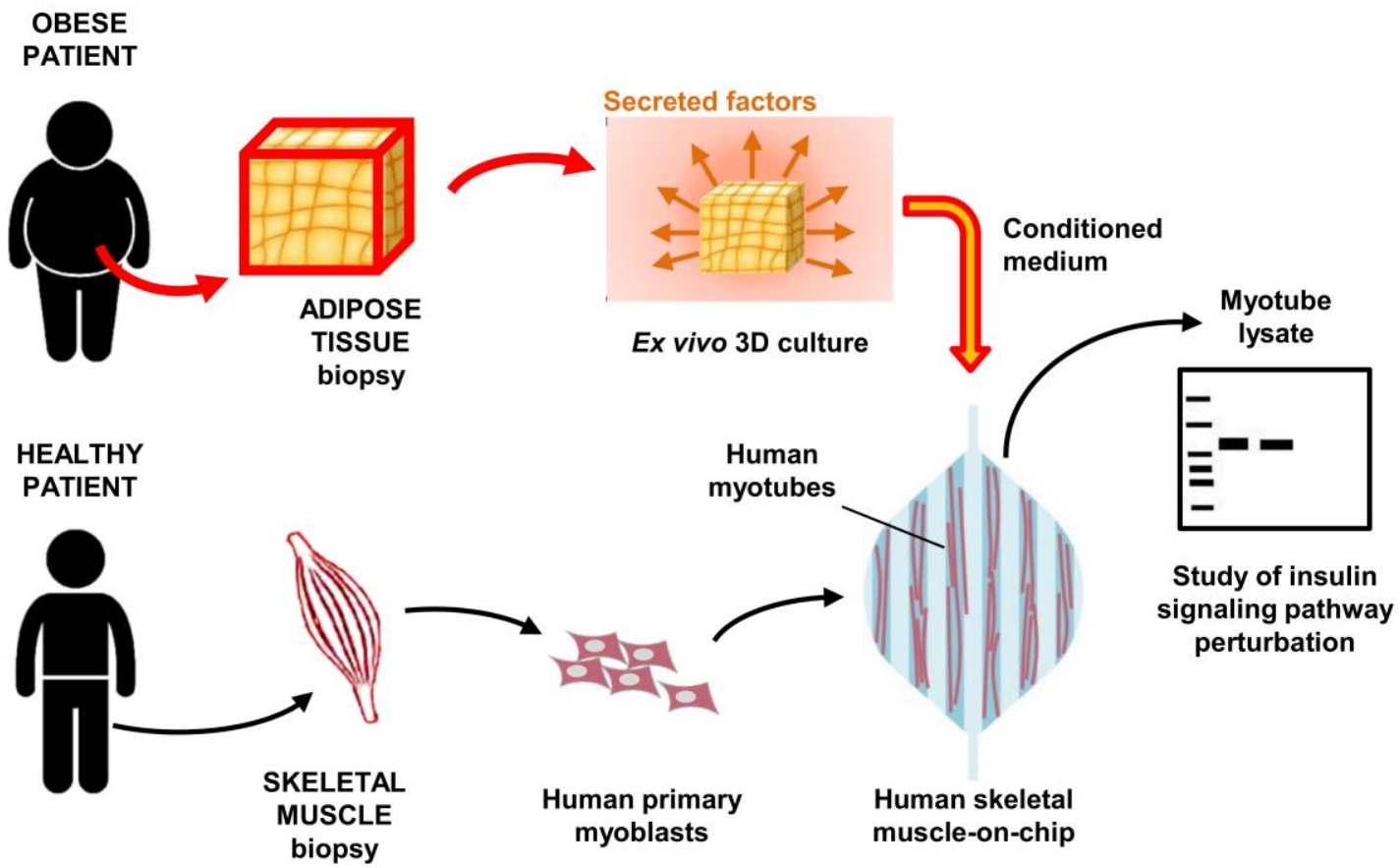

Figure 1. Study of the cross-talk between an adipose tissue from an obese patient and a skeletal muscle from a lean patient. A biopsy of human adipose tissue was maintained in a threedimensional culture ex vivo for 48 hours. A human skeletal muscle-on-chip model was developed from human primary myoblasts, which were induced to differentiate into mature myotubes within a microfluidic chamber by a combination of exogenous factors in the culture medium, concentrated cell secreted factors and surface topology control. 

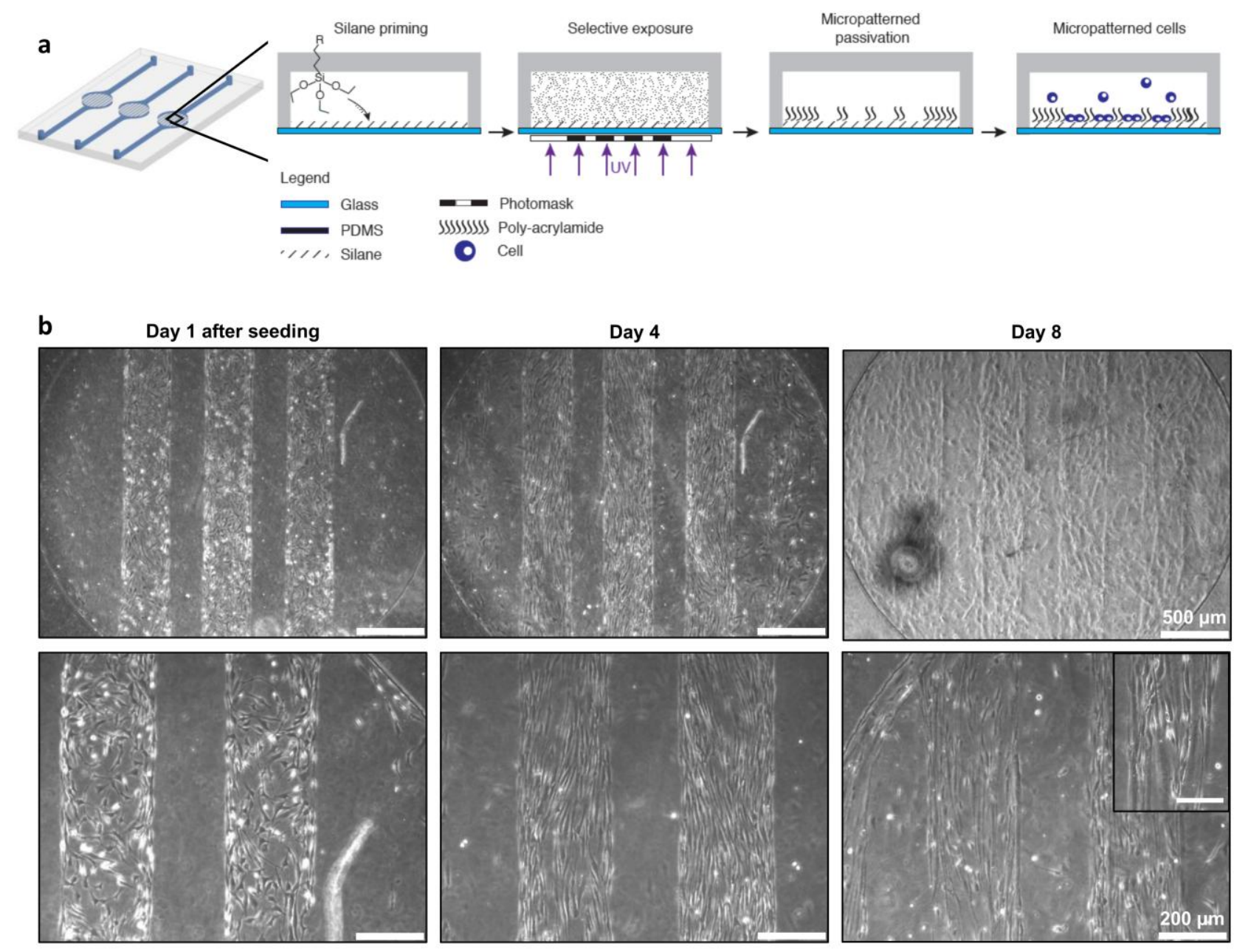

Figure 2. Human skeletal-muscle on chip development. (a) Schematic of adhesive pattern production and functioning. (b) Temporal evolution of human primary myoblast morphology within the patterned microfluidic culture chamber. Scale bars: $500 \mu \mathrm{m}$ (top row), $200 \mu \mathrm{m}$ (bottom row), and $100 \mu \mathrm{m}$ (enlargement). 


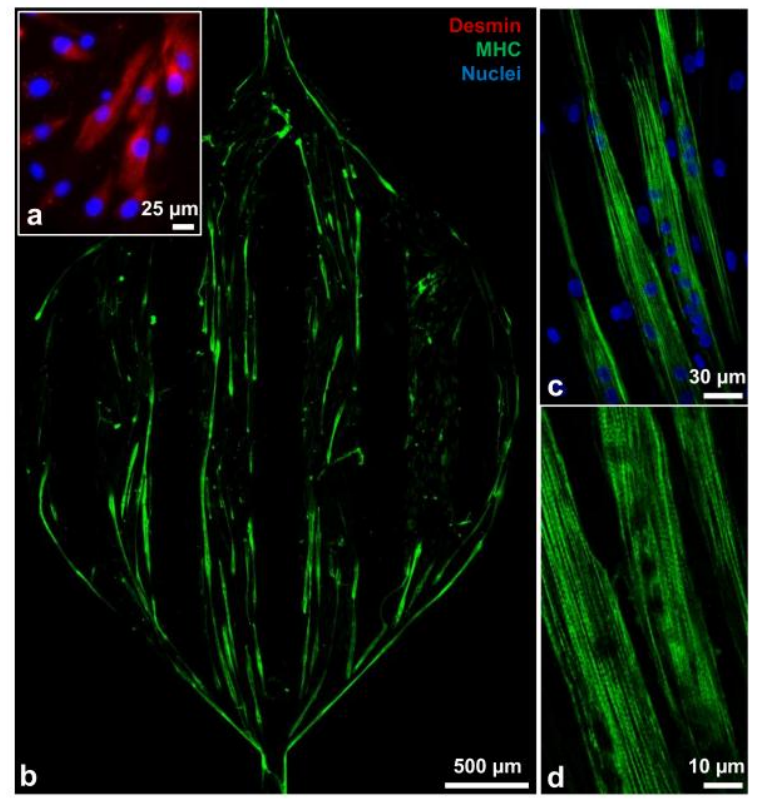

Figure 3. Characterization by immunofluorescence of myotubes within the skeletal muscle-on-chip system. (a) Desmin and nuclei staining. (b) Myosin heavy chain (MHC) staining of a whole patterned culture chamber. (c-d) Enlargement from (b), highlighting the skeletal myotube sarcomere organization into a striated regular pattern, with and without nuclear staining. 

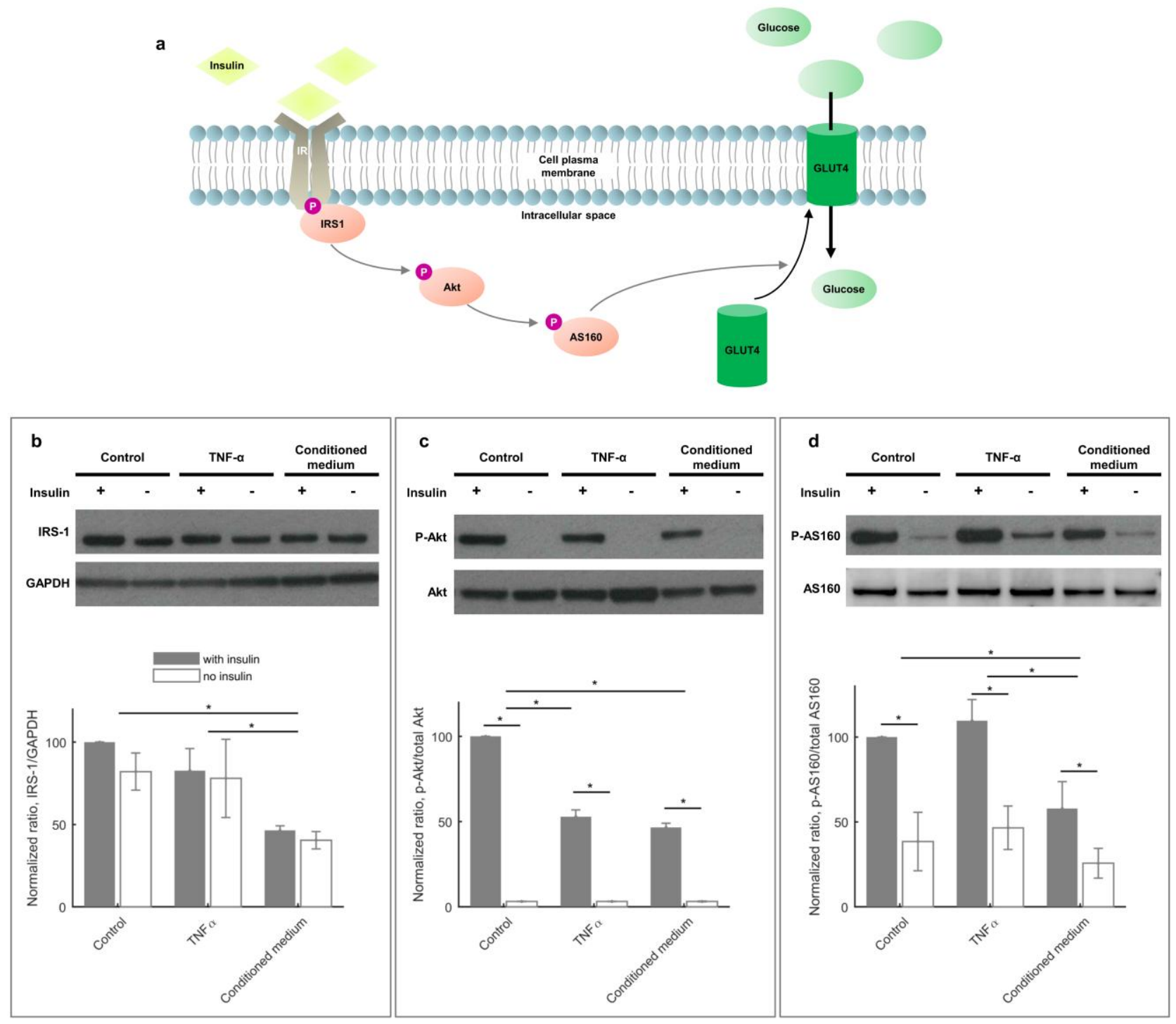

Figure 4. Results of the cross-talk between a human adipose tissue from an obese patient and a skeletal muscle from two healthy patients. (a) Schematic representation of insulin signaling pathway in a skeletal muscle cell, limited to the components of the pathway measured in this study: extracellular insulin binds to the insulin receptor (IR) and activates a phosphorylation cascade that ultimately induces the translocation of the glucose transporter GLUT4 to the cell plasma membrane. The main physiological effect of this pathway is the insulin-stimulated uptake of glucose by the skeletal muscle. (b-d) Quantification, by Western blot analysis, of insulin signaling-related proteins in myotubes after a 24-hour pre-conditioning with TNF $\alpha$ or adipose tissue-conditioned medium, and a 10-minute insulin stimulation. 